\title{
EFECTO DEL PRECALENTAMIENTO EN AGUA SOBRE LA DENSIDAD BÁSICA Y LA CONTRACCIÓN EN COIGÜE
}

Nothofagus dombeyi. Rubén A. Ananías, Profesor Asistente, Depto. Ing. Maderas, Facultad Ingeniería, Universidad del Bío-Bío. Casilla 5-C, Concepción, Chile. Marcela Chamorro y Carmen Díaz, Ingenieros Ejecución en Maderas, INFODEMA S.A. Casilla 732, Valdivia, Chile.

\section{RESUMEN}

En este trabajo se analizó el comportamiento de la densidad básica y la contracción de muestras de coigüe Nothofagus dombeyi de $30 \mathrm{~mm} \times 30 \mathrm{~mm} \times 300 \mathrm{~mm}$, frente al precalentamiento prolongado en agua caliente.

El diseño experimental incluyó como factores variables el tiempo de calentamiento en cuatro niveles $\left(4,812\right.$ y 16 dias) y la temperatura de calentamiento en tres niveles $\left(40,75\right.$ y $\left.95^{\circ} \mathrm{C}\right)$ y se contemplaron 3 repeticiones por tratamiento. Un lote de muestras sin calentamiento fue utilizado como control.

Los resultados indican que la densidad básica decrece en forma lineal con el tiempo de calentamiento y se detectó más de un $12 \%$ de pérdida de la densidad básica con temperatura de calentamiento superior $a 75^{\circ} \mathrm{C}$. La contracción total aumentó significativamente con el tiempo de calentamiento y la temperatura debido al mayor colapso en la madera pretratada.

Palabras clave: Nothofagus dombeyi; Colapso; Contracción; Densidad; Precalentamiento; Secado; Chile.

\section{ABSTRACT}

We heated samples of chilean coigüe Nothofagus dombeyi in water and studied the effect on basic density and shrinkage. Our samples measured $30 \mathrm{~mm} \times 30 \mathrm{~mm} \times 300 \mathrm{~mm}$.

We used four heating times $(4,8,12$ and 16 days) and three water temperatures $(40,75$ and $\left.95^{\circ} \mathrm{C}\right)$, with three replications each and control samples.

Basic density decreased linearly with heating time. There was a 12-percent basic density loss with temperatures $75^{\circ} \mathrm{C}$ and higher. Shrinkage increased with heating time and temperature, due to an increase in collapse.

Keywords: Nothofagus dombeyi; Collapse; Shrinkage; Density; Preheating; Drying; Chile. 


\section{INTRODUCCIÓN}

Diversas formas de pretratamientos se han estado empleando para mejorar el comportamiento de la madera aserrada frente al secado y otros procesos de transformación mecánica de la madera, en particular para el coigüe, Nothofagus dombeyi, además se persigue homogeneizar el color de la madera. Los fluidos empleados con más frecuencia en los pretratamientos han sido: vapor saturado, agua caliente y ambiente saturado. La temperatura y el tiempo de tratamiento dependen del objetivo perseguido con el pretratamiento.

Evidencias del aumento de la velocidad del secado en madera pretratada con vapor saturado por corto tiempo ( 2 a 4 horas) han sido reportadas para especies del género Eucalyptus y Nothofagus (Campbell, 1961; Haslett y Kininmonth, 1986; Alexiou et al., 1990; Chafe y Ananías, 1996). Similar comportamiento de la tasa de secado en red-beech Nothofagus fusca, ha mostrado el pretratamiento de 2 horas con agua caliente a $70{ }^{\circ} \mathrm{C}$ (Haslett y Kininmonth, 1986) y el eucalipto Eucalyptus globulus pretratada por 3 horas en vapor húmedo a $80^{\circ} \mathrm{C}$ (Ananías et al., 1995a). Este mejoramiento de la velocidad del secado frente al pretratamiento ha sido asociado a cambios químico-físicos en la madera, por una parte a un efecto de reubicación de las depositaciones de los lúmenes celulares y por otra parte a una leve hidrólisis ácida de la pared celular (Mackay, 1971).

Otra implicancia del pretratamiento, ha sido su efecto sobre las propiedades anatómicas, físicas y mecánicas de la madera.

Ciertas depositaciones en la pared celular y en las punteaduras de las células de almacenamiento ha sido reportado en duramen de eucalipto Eucalyptus pilularis (Alexiou et al., 1990). Adicionalmente se ha observado un mejor comportamiento frente a la recuperación del colapso de Eucalyptus pilularis prevaporizada por media hora a $100^{\circ} \mathrm{C}$ (Alexiou et al., 1990). También se ha observado un tercio más recuperación del colapso en Eucalyptus regnans precalentada en agua hirviente por 8 minutos (Chafe, 1993), además se ha detectado un menor colapso y mayor propensión a la recuperación del colapso en madera pretratada de Eucalyptus globulus (Ananías et al., 1995a).

Se ha encontrado una correlación negativa entre la densidad de la madera y la propensión a las grietas internas y en la recuperación del colapso de la madera pretratada. La misma tendencia es observada para el contenido de humedad inicial y la propensión a las grietas internas de la madera (Chafe, 1994). El excesivo colapso y las grietas internas durante el secado de la madera pretratada, provienen de una leve hidrólisis ácida de la pared celular de las fibras (Mackay, 1971). Los pretratamientos prolongados a temperaturas convencionales, han mostrado evidencias de aumento de la contracción y del colapso en eucalipto Eucalyptus regnans (Kauman, 1961), de grado más pronunciado de la calidad de la madera de red-beech Nothofagus fusca durante el secado (Haslett y Kininmonth, 1986) y una reducción en las propiedades de resistencia 
mecánica en flexión del coigüe Nothofagus dombeyi (Ananías et al., 1995b). No obstante, se observó mayor velocidad del secado y un menor indice de defectos de secado del coigüe Nothofagus dombeyi precalentado en agua caliente por largo tiempo en el rango de temperatura de 40 a $60^{\circ} \mathrm{C}$ (Díaz-Vaz y Poblete, 1991).

En este trabajo se analiza el precalentamiento prolongado en agua de madera verde de coigüe Nothofagus dombeyi y su impacto sobre la densidad básica y la contracción del material.

\section{METODOLOGÍA}

La madera fue recogida recién aserrada en una planta industrial de la Décima Región de Chile. Se seleccionaron 12 piezas verdes (recién aserradas) de $42 \mathrm{~mm}$ x $250 \mathrm{~mm}$ x $3600 \mathrm{~mm}$, proveniente de igual número de trozas (árboles). Tales piezas fueron cortadas en muestras de dimensión $30 \mathrm{~mm}$ x $30 \mathrm{~mm}$ x $300 \mathrm{~mm}$ y luego divididas al azar una para cada combinación de temperatura y tiempo.

El plan experimental fue una combinación de 4 tiempos de precalentamiento $(4,8,12$ y 16 días) y tres temperaturas $\left(40,75\right.$ y $\left.95^{\circ} \mathrm{C}\right)$, con tres repeticiones por tratamiento. Un lote de muestras fue dejado sin pretratamiento para control.

El tratamiento fue llevado a cabo en un baño termostático y la temperatura del agua se mantuvo constante agitando el agua en el recipiente. Por cada tratamiento se introdujeron en el baño las muestras verdes para 16 días y luego fueron agregados los lotes de muestras con tiempos menores de tratamiento, hasta el último lote de 4 días. Para mantener el contenido de humedad original (verde) las muestras fueron envueltas en bolsas de polietileno y dejadas en una sala de clima fría, a la espera de su turno de inmersión.

Antes y después de cada precalentamiento fueron medidos los pesos y las dimensiones de las muestras. Posteriormente las muestras fueron acondicionadas en una cámara de clima Heraeus-Votsch a $30^{\circ} \mathrm{C}$ y un contenido de humedad de equilibrio de $12 \%$.

En esta condición, cuando la madera alcanzó el peso constante, se midieron nuevamente los pesos y las dimensiones de las muestras. Además se recogieron pequeñas probetas (10 mm arista) para la obtención de cortes en los tres planos de referencia y observar al microscopio electrónico la estructura de la madera. Finalmente todas las muestras fueron secadas en estufa a $103^{\circ} \mathrm{C}$, hasta peso anhidro.

Microfotos de las muestras tomadas fueron obtenidas bajo el microscopio electrónico. Análisis de varianza y de regresión lineal fue realizado para evaluar el efecto del tiempo y de la temperatura del calentamiento sobre la densidad básica y la contracción de la madera. 


\section{RESULTADOS Y DISCUSIÓN}

La Figura 1 muestra las microfotografías de la estructura anatómica de la madera de coigüe ensayada. El efecto sobre la anatomía de la madera comienza a evidenciarse a temperaturas de $75{ }^{\circ} \mathrm{C}$ y por un tiempo prolongado a 16 días. Por debajo de esta condición no parece presentarse un cambio notable en la composición de las fibras de la madera. Se aprecia, un leve aplastamiento de las células en la dirección tangencial para un pretratamiento de $40^{\circ} \mathrm{C}$ y 16 días de inmersión (Figura lb)
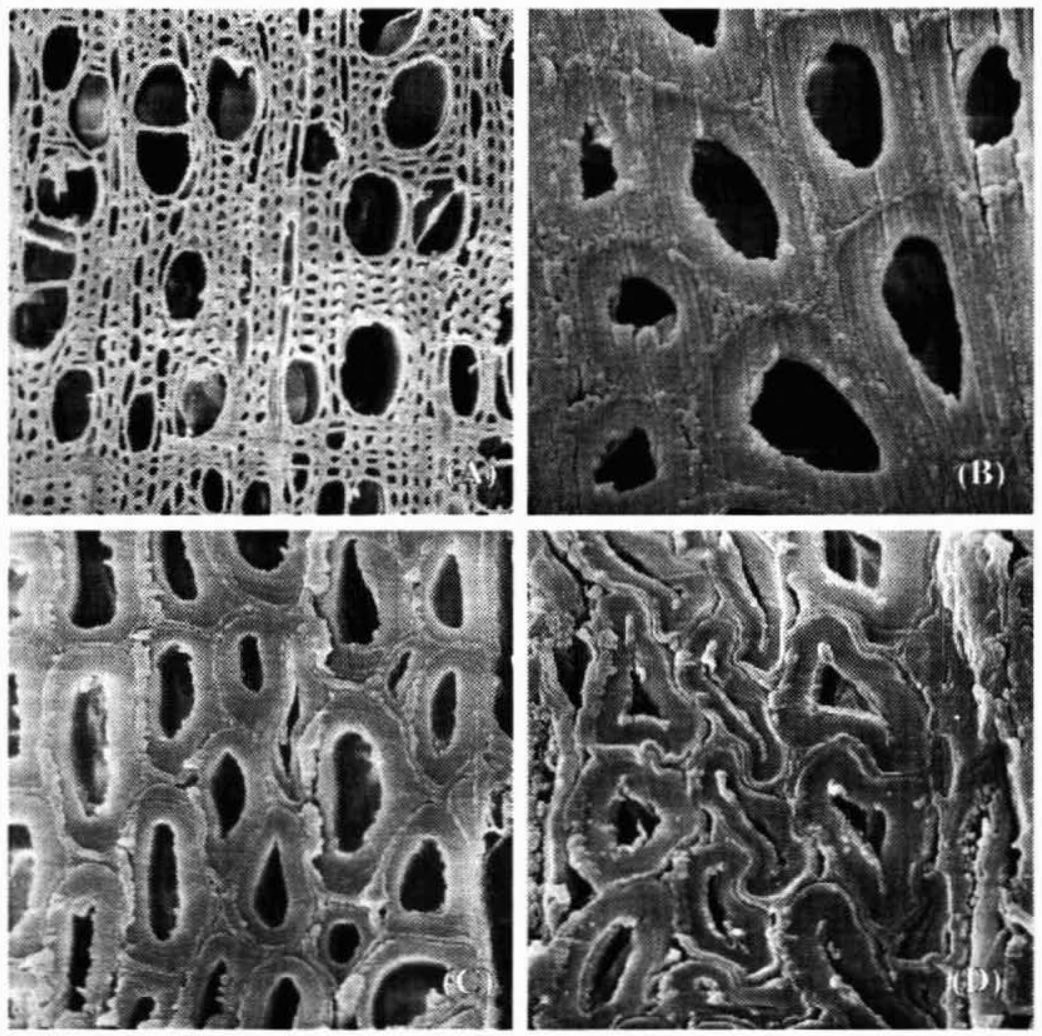

Figura 1. MICROFOTOGRAFÍAS COIGÜE Nothofagus dombeyi. (A) SECCIÓN TRANSVERSAL COIGÜE CONTROL (180X); (B) SECCIÓN TRANSVERSAL COIGÜE PRECALENTADO A $40^{\circ} \mathrm{C}$ POR 16 DÍAS (2500X); (C) SECCIÓN TRANSVERSAL COIGÜE

PRECALENTADO EN AGUA CALIENTE A $75^{\circ} \mathrm{C}$ POR 16 DÍAS (800X); (D) SECCIÓN

TRANSVERSAL COIGÜE PRECALENTADO EN AGUA CALIENTE A $95^{\circ} \mathrm{C}$ POR 12 DÍAS (800X). (FOTOGRAFÍAS:GENTILEZA UNIVERSIDAD DE CONCEPCIÓN) 
Con $75^{\circ} \mathrm{C}$ para 16 días se alcanza una importante alteración de los tejidos leñosos (Figura 1c). Ello es manifestado por una degradación a nivel de la lamela compuesta (lamela media más pared primaria). Probablemente el efecto del calentamiento en esta zona sea favorecido por el pequeño espesor de esta lamela, la reducida presencia de microfibrillas de celulosa y la abundancia de lignina en esta región de las fibras. La presencia de agua y temperatura favorece la deformación plástica de la madera. Cuando el precalentamiento es más severo (condición $95^{\circ} \mathrm{C}$ y 12 días), se observa una deformación intensa de la pared celular, la que es potenciada por la carencia de lamela media compuesta que ha sido degradada enteramente (Figura 1d). En esta condición la madera ha reducido a un cuarto su resistencia mecánica original (Ananías et al., 1995b).

Este comportamiento de la pared celular de las fibras, puede ser atribuido degradación térmica de la pared celular de las fibras, similar a lo reportado para el precalentamiento de Eucalyptus regnans a temperatura elevada y de larga duración o por alta temperatura de corta duración (Kauman, 1961). Ésta se intensifica en presencia de agua caliente a temperaturas elevadas por tiempos prolongados y con la madera verde. Ello se evidencia cuando la pérdida de la densidad básica fue apreciable a $95^{\circ} \mathrm{C}$ y 12 días de inmersión (Figura 2), la densidad básica cayó desde aproximadamente 0,52 $\mathrm{g} / \mathrm{cm} 3$ en la madera control a $0,46 \mathrm{~g} / \mathrm{cm} 3$ en la madera pretratada.

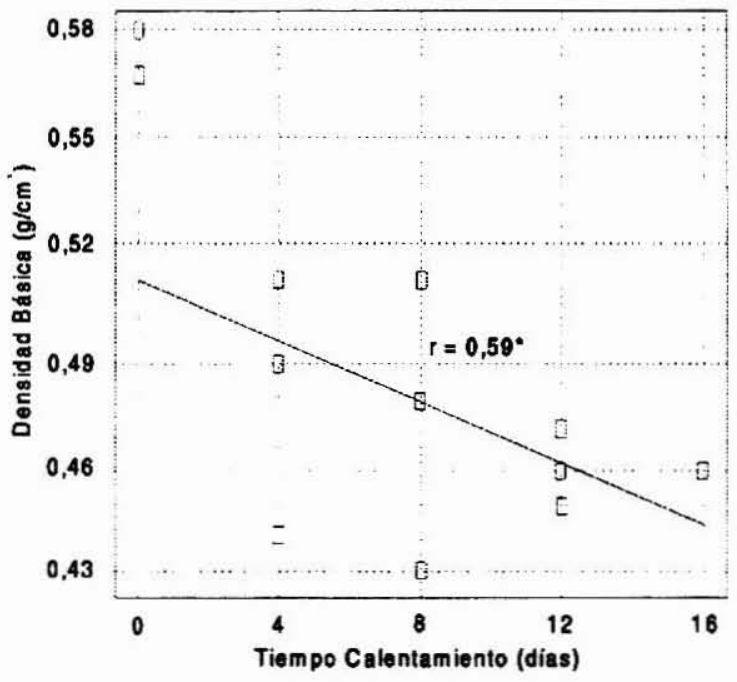

Figura 2. CORRELACIÓN ENTRE DENSIDAD BASICA Y TIEMPO CALENTAMIENTO EN COIGÜE NOTHOFAGUS DOMBEYI PRECALENTADO EN AGUA CALIENTE A $95^{\circ} \mathrm{C}$. ( $r$ = COEFICIENTE DE CORRELACIÓN; * = CORRELACIÓN SIGNIFICATIVA, SEGÚN T-TEST) 


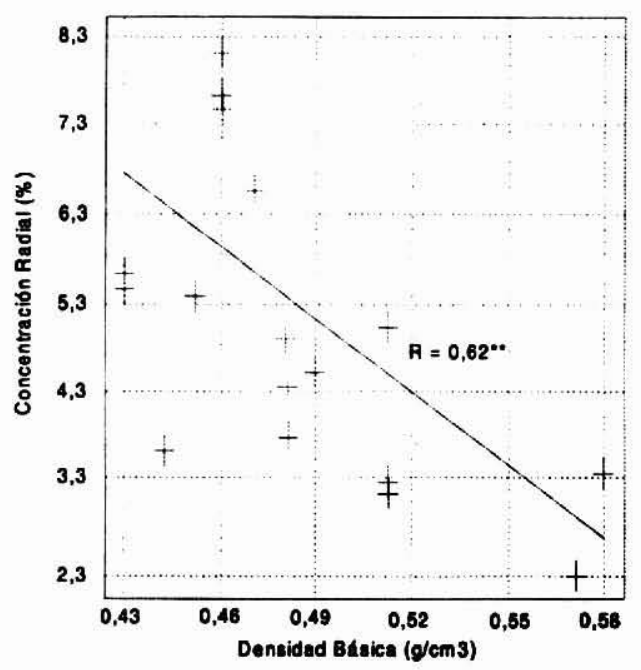

Figura 3. CORRELACIÓN ENTRE CONTRACCIÓN RADIAL Y DENSIDAD BÁSTCA EN COIGÜE Nothofagus dombeyi PRECALENTADO EN AGUA CALIINTTE A $95^{\circ} \mathrm{C}$.

(R = COEFICIENTE DE CORRELACIÓN; ** = CORRELACIÓN MUY SIGNIFICATIVA, SEGÚN TTEST).

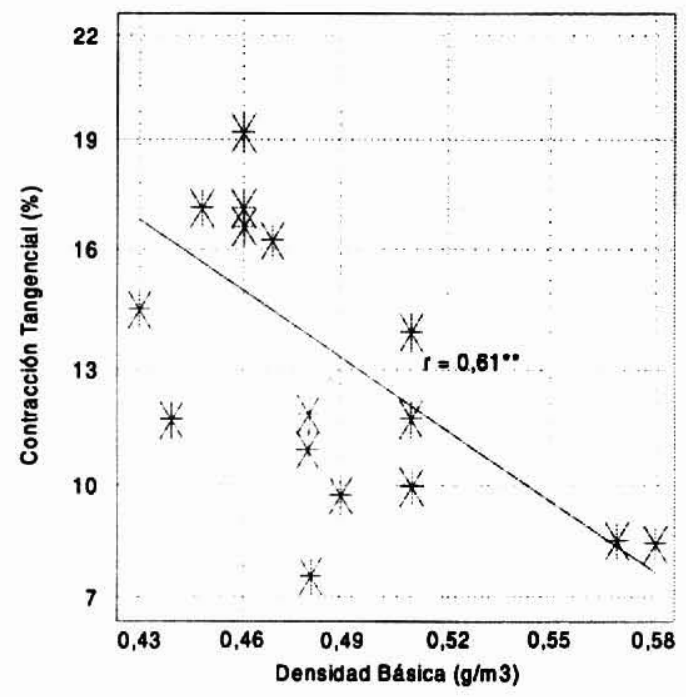

Figura 4. CORRELACIÓN ENTRE CONTRACCIÓN TANGENCIAL Y DENSIDAD BÁSICA EN COIGÜE Nothofagus dombeyi PRECALENTADO EN AGUA CALIENTE A $95^{\circ} \mathrm{C}$.

$(R$ = COEFICIENTE DE CORRELACIÓN; ** = CORRELACIÓN MUY SIGNIFICATIVA, SEGÚN TTEST). 
Esta disminución en cerca de un $12 \%$ de la densidad básica del coigüe, que no es estadísticamente significativa, tal como se aprecia en el Cuadro 1 deviene fundamentalmente de una pérdida de peso de la sustancia leñosa. Tal comportamiento de la densidad básica trae como consecuencia un aumento en la contracción radial (Figura 3) y contracción tangencial (Figura 4).

De acuerdo al Cuadro 1, la temperatura y el tiempo de calentamiento afectan muy significativamente a la contracción radial y tangencial. Por otra parte en las Figuras 5 y 6 se aprecia que el tiempo y la temperatura favorecen los cambios dimensionales de la madera en el sentido radial y tangencial, respectivamente. Este impacto es más notable a temperatura de $75^{\circ} \mathrm{C}$ o superior. La madera control alcanza una contracción de $2,9 \%$ y $8,4 \%$ en dirección radial y tangencial, la cual es mantenida hasta un pretratamiento de 75 ${ }^{\circ} \mathrm{C}$ y 8 días, por más tiempo o incrementando la temperatura la contracción incrementa a $8 \%$ y $20 \%$ en dirección radial y tangencial a los $95^{\circ} \mathrm{C}$ y 16 días de precalentamiento. Este incremento de la contracción total después de los 8 días de ensayo a $75^{\circ} \mathrm{C}$, es ocasionado fundamentalmente por el aumento del colapso de la madera, potenciado por la degradación térmica de la pared celular de las fibras.

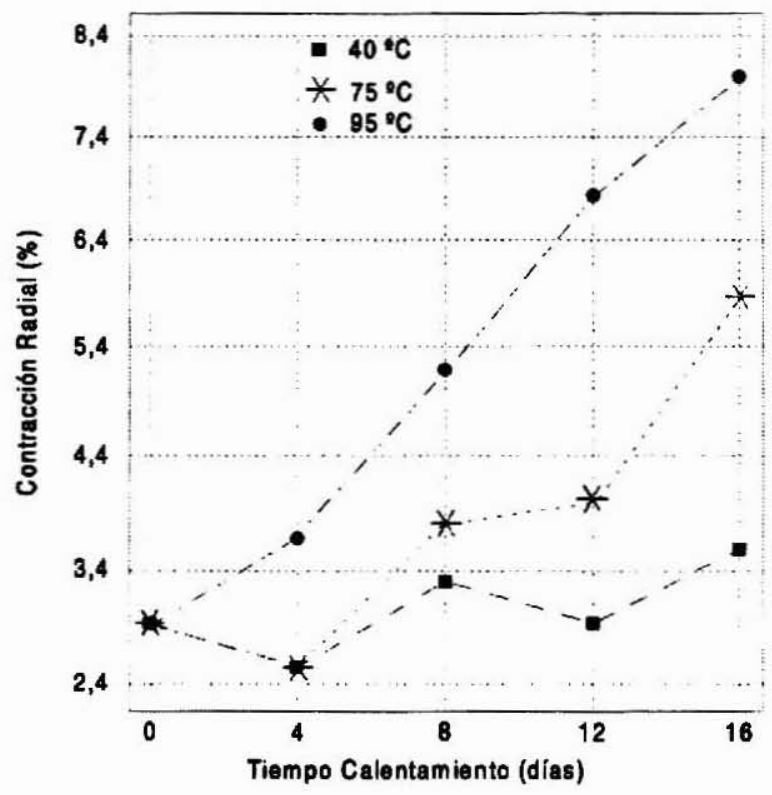

Figura 5. RELACIÓN ENTRE CONTRACCIÓN RADIAL Y TIEMPO CALENTAMIENTO A DISTINTAS TEMPERATURAS EN COIGÜE Nothofagus dombeyi PRECALENTADO EN AGUA CALIENTE. 


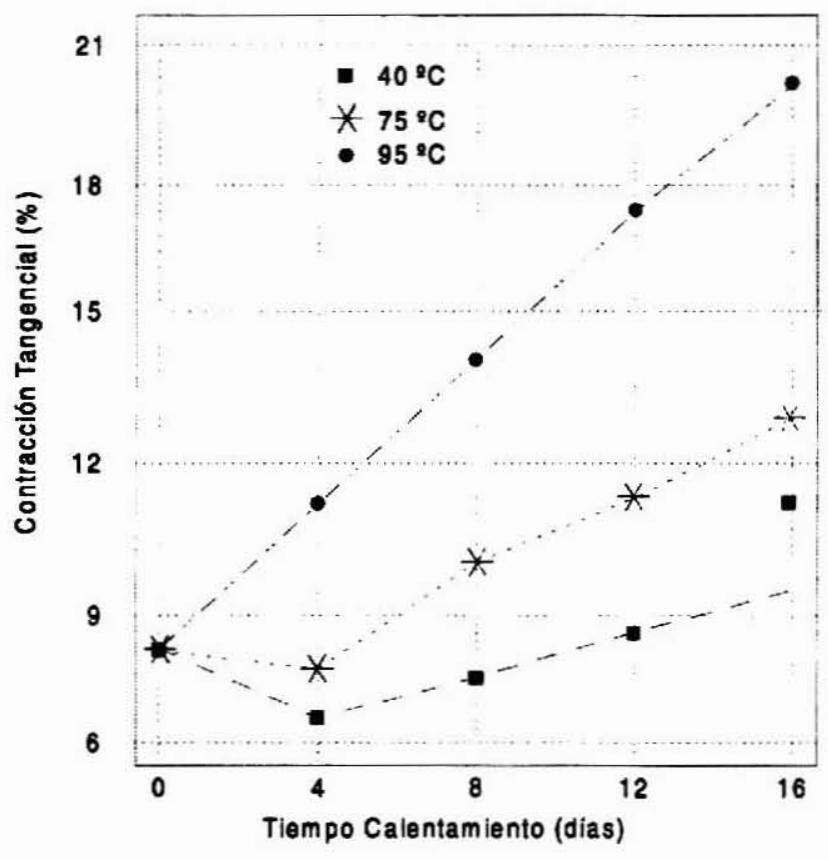

Figura 6. RELACIÓN ENTRE CONTRACCIÓN TANGENCIAL Y TIEMPO DE CALENTAMIENTO A DISTINTAS TEMPERATURAS EN COIGÜE Nothofagus dombeyi PRECALENTADO EN AGUA CALIENTE.

Cuadro 1

VALORES DE PROBABILIDAD (P-VALUE) SEGÚN ANÁLISIS DE VARIANZA (F-TEST).

\begin{tabular}{|c|c|c|c|}
\hline \multirow{2}{*}{ Factor } & \multirow{2}{*}{ Densidad Básica } & \multicolumn{2}{|c|}{ Contracción } \\
\cline { 3 - 4 } & NS & Radial & Tangencial \\
\hline Tiempo & NS & 0,030 & 0,0010 \\
\hline Temperatura & N & 0,008 & 0,0002 \\
\hline
\end{tabular}

P-value $<0,05$ implica diferencia significativa.

$P$-value $<0,01$ implica diferencia muy significativa.

NS $=$ No Significativa.

\section{CONCLUSIONES}

El precalentamiento prolongado en agua caliente a $75^{\circ} \mathrm{C}$ aumenta significativamente la contracción total radial y tangencial del coigüe. Este incremento se debe a una degradación térmica de la lamela media compuesta en la pared celular de las fibras, lo que favorece el colapso de la madera. 
La densidad básica disminuye pero no significativamente, con el precalentamiento prolongado en agua caliente del coigüe. Tal disminución es ocasionada por la pérdida de peso de la sustancia leñosa debido a la degradación de la lamela media compuesta en la pared celular de las fibras.

\section{REFERENCIAS}

Alexiou, P.N.; Wilkins, A.P.; Hartley, J. 1990. Effect of Presteaming on Drying Rate, Wood Anatomy and Shrinkage of Regrowth Eucalyptus pilularis. Wood Sci.Technol. 24:103-110.

Ananías, R.A.; Espinoza, L.; Kauman, W.G. 1995a. Preheating of Chilean Eucalyptus globulus. Holzforschung 49(2): 179-181.

Ananías, R.A.; Chamorro, C.; Díiaz, C. 1995b. Some Effects of Heating Chilean Coigüe in Water. Poster IUFRO XX World Congress, Tampere, Finland.

Campbell, G.S.1961. The Value of Presteaming for Drying some Susceptible Eucalypts. Forest Prod. J. 11(8):343-347.

Chafe, S.C.; Ananías, R.A. 1996. Effect of Presteaming on Moisture Loss and Internal Checking in High-Temperature-Dried Boards of Eucalyptus globulus and Eucalyptus regnans. J. Inst. Wood Science (in press).

Chafe, S.C. 1994. Preheating Green Boards of Eucalyptus regnans. II Relationships amongst Properties. Holzforschung 48(2):163-167.

Chafe, S.C. 1993. The Effect of Boiling on Shrinkage, Collapse and other Wood-Water Properties in Core Segments of Eucalyptus regnans. Wood Sci. Technol. 27:205-217.

Chafe, S.C. 1990. Effect of Brief Presteaming on Shrinkage, Collapse and other Wood Water Relationships in Eucalyptus regnans. Wood Sci. Technol. 24:311-326.

Díaz-Vaz, J.E.;Poblete, H. 1991. Inmersión en Agua de Madera Aserrada de Coigüe. Actas VII Reunión Investigación en Productos Forestales 1:241-259. Valdivia, Chile.

Haslett, A,.N.; Kininmonth, J.A. 1986. Pretreatments to Hasten the Drying of Nothofagus fusca. New Zealand J. Forestry Sci. 16(2):237-246.

Kauman, W.G. 1961. Effect of Thermal Degradation on Shrinkage and Collapse of Wood from 3 Australian Species. Forest Prod. J. 11(9):445-452.

Mackay, J.F. 1971. Influence of Steaming on Water Vapor Diffusion in Hardwoods. Wood Sci. 16:48-56. 